\title{
Splenogonadal Fusion, Rare Cause of Scrotal Mass in Children: A Case Report \\ ${ }^{1}$ Muhammad Ali Sheikh, ${ }^{1}$ Fatima Malik, ${ }^{2}$ Sadaf Batool, ${ }^{1}$ Jamil Akhtar Munir Ahmed, ${ }^{1}$ Tariq Latif \\ ${ }^{1}$ Department of Paediatric Surgery, Shaikh Zayed Hospital, Lahore \\ ${ }^{2}$ Departmnet of Surgical Oncology, Shaukat Khanum Hospital, Lahore
}

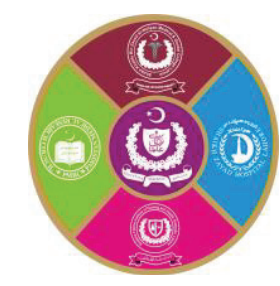

\begin{abstract}
Introduction: Splenogonadal fusion (SGF) is a rare congenital malformation which is caused by an abnormal fusion between the primitive spleen and developing gonad. SGF has been classified into two types. Continuous type, where there is a connection between the spleen and the gonad; and discontinuous type, where ectopic part of spleen is attached to the gonad but there is no connection with the orthotopic spleen. Patients with continuous SGF frequently have additional congenital anomalies most common of which is cryptorchidism and is present in almost $31 \%$ of patients along with inguinal hernias.

Case Presentation: We report a 3 years old child who was operated for right inguinal hernia and left communicating hydrocele and on exploration was found to have left sided continuous type splenogonadal fusion. He underwent excision of fused part of spleen along with left orchiectomy because of abnormal looking testis.

Conclusion: SGF is a rare entity mostly unknown to surgical community. Orchidectomy should be avoided in these cases. It is often associated with other congenital anomalies.
\end{abstract}

Key words: Splenogonadal Fusion, Inguinal hernia, Scrotal mass children.

\section{INTRODUCTION}

$\mathrm{S}$ 200 cases reported to date. More common in males due to superficial location of male gonads as compared to female gonads. About $80 \%$ of cases are diagnosed before the age of 10 years. ${ }^{1}$ It is more prevalent in Caucasian population followed by African origin, ${ }^{2}$ Exact etiology of SGF is not known. It may result from fusion of spleen with peri-mesonephros. It may be of continuous or discontinuous type but continuous variety is more common. $^{3}$ Splenic tissue in SGF is normal and orchidectomy and splenectomy is not indicated. In our reported case, orchidectomy was performed because the surgeon suspected it to be a malignancy.

\section{CASE REPORT}

A 3-years old male child was admitted in Pediatric Surgery ward, through outpatient department on August 2017 with bilateral scrotal swelling from last six months that was increasing gradually in size. It was associated with occasional episodes of mild, dull aching pain that was aggravated on exertion. No history of fever, constipation, chronic cough was noted. On local examination, patient had bilateral scrotal swelling without any overlying skin changes or scar marks. On right side there was a $4 \mathrm{x} 4 \mathrm{~cm}$ soft non-tender reducible swelling with positive cough impulse. It was not possible to get above the swelling and right testis was separately palpable. On left side, there was a $3 \times 3 \mathrm{~cm}$ soft swelling which was neither tender nor reducible and left testis was not separately palpable but swelling decreased in size on lying down. On the basis of clinical examination, diagnosis of right inguinal hernia and left communicating hydrocele was made. Preoperative plan was to do right herniotomy and left patent processes vaginalis ligation. Preoperative optimization was done with baseline investigations including complete blood count and urea and electrolytes followed by anesthesia evaluation. Peroperatively right herniotomy was done by right inguinal skin crease incision as planned. Then left side was explored during which abnormal looking tissue was observed in left inguinal canal (Fig-1). There was a reddish brown, thick cord like structure 
fused with testis, extending through deep inguinal ring into the abdomen (Fig-2).

Exploratory laparotomy was done through left transverse lower abdominal incision to trace site of origin of that abnormal looking cord like structure due to high suspicion of malignancy. It was not possible to separate the hernia sac from this tissue. The tissue fused with left testis was found extending up to lower pole of spleen.

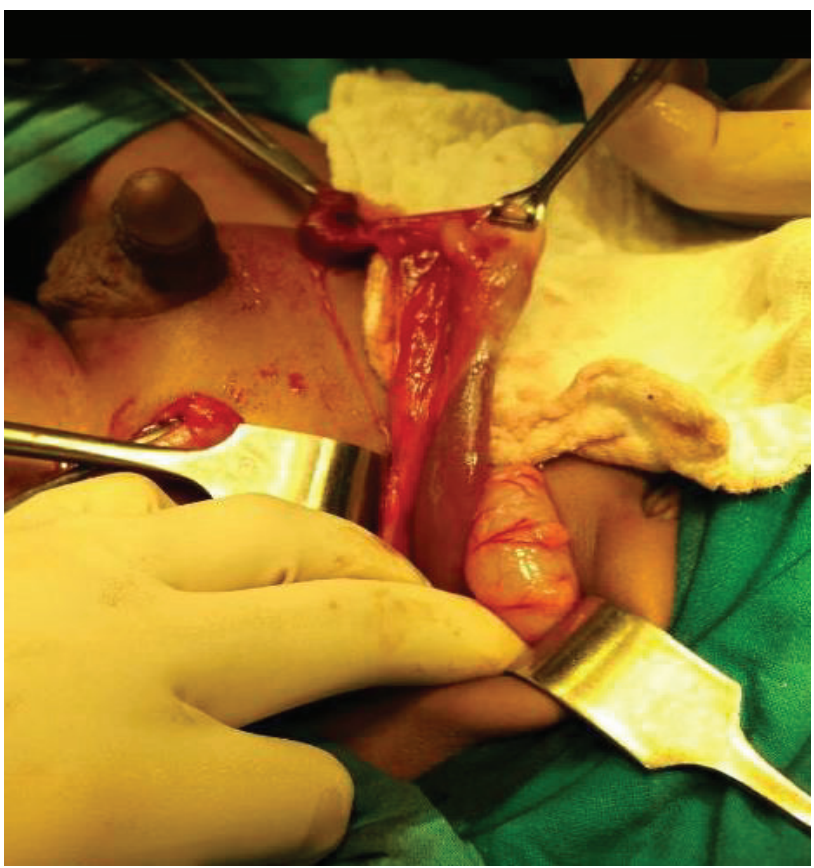

Fig-1: Intraoperative picture showing cord like splenic tissue and left testis

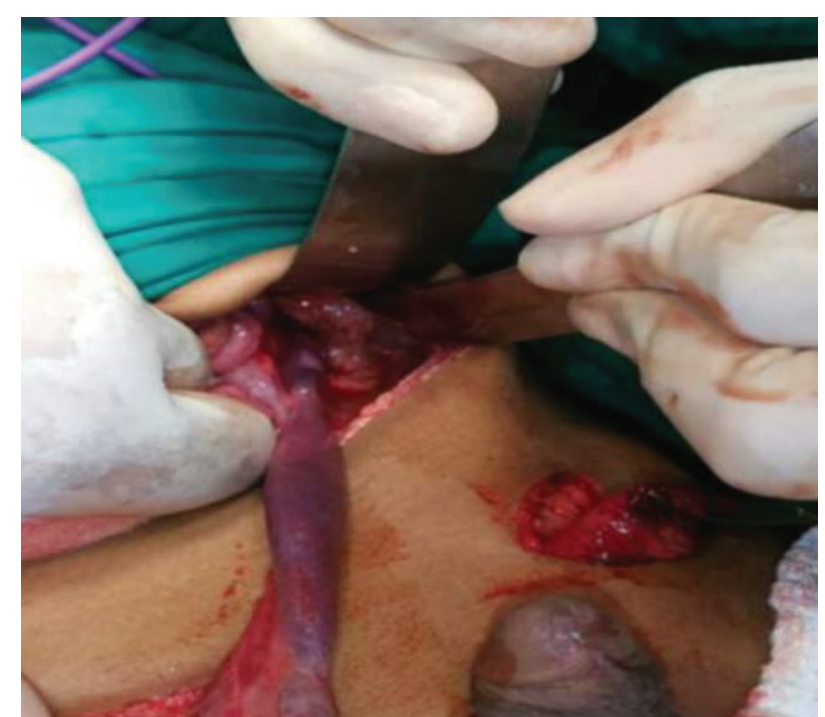

Fig-2: Intraoperative picture showing splenic tissue extending into abdomen.

Because of abnormal looking mass arising from left testis and non-availability of frozen section, left orchidectomy was performed along with excision of fused tissue up to lower pole of spleen. Specimen was sent en block for histopathology (Fig-3). Patient remained vitally stable post operatively and was discharged after 3 days on oral analgesics and antibiotics. On his first follow up visit after 1 week, patient was found to be recovering well with healing wound. Histopathology report revealed benign splenic tissue, testis, epididymis, vas deferens and spermatic cord consistent with splenogonadal fusion.

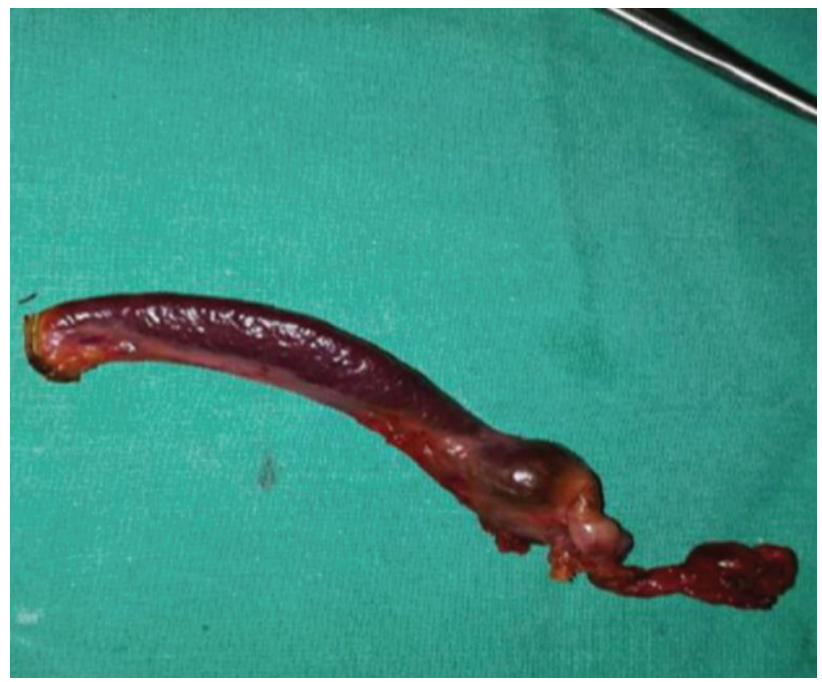

Fig-3: Resected specimen showing splenic tissue and attached gonad

\section{DISCUSSION}

Splenogonadal fusion (SGF) is an uncommon entity and is mostly diagnosed incidentally. Cryptorchidism is most common reason for exploration. Other associated anomalies include limb defects, cardiac anomalies, craniosynostosis, anal anomalies and Moebius syndrome. ${ }^{4,5}$ Although SGF is commonly associated with other anomalies, our patient had no other associated anomalies. It usually presents as testicular mass or inguinal hernia. ${ }^{1}$ If discontinuous, testicular swelling may be the presentation, appearing as hydrocele. ${ }^{6}$ In our case, patient presented as left communicating hydrocele. A major factor in its misdiagnosis is the lack of awareness of SGF. It is mostly diagnosed intraoperatively but Doppler ultrasound of scrotum has been used to diagnose a few cases of SGF. ${ }^{7}$ Technetium 99 scan is very useful for continuous variety of SGF. ${ }^{8}$ Magnetic resonance imaging (MRI) is performed rarely but it confirms the splenic origin of the lesion. ${ }^{9}$ These investigations are helpful if there is preoperative suspicion of SGF. Otherwise the diagnosis of SGF prior to surgery is challenging. SGF usually presents as an asymptomatic testicular swelling. Other 
manifestations may include acute testicular pain and swelling caused by ectopic splenic tissue infections. The decision to resect the entire splenogonadal tissue in our case was made upon the abnormal look of the testis, its fusion to ectopic splenic tissue and inability to separate hernia sac from the fused tissue. No surgery is required if an accurate diagnosis could be made pre-operatively specially in cases of discontinuous variety. The testis may be preserved in most cases even if surgery is performed. Laparoscopy is very helpful in doubtful cases of continuous variety. Splenic tissue can be separated from the gonad and as a result the testis may be preserved. If a tumor is suspected, frozen sectioning may aid with the diagnosis.

Due to benign nature of splenic tissue, excision is not recommended in discontinuous type and it is better left alone if diagnosis is confirmed preoperatively. Orchidectomy is mostly performed due to lack of awareness about this condition and hard consistency of tissue leading to suspicion of underlying malignancy but intra-operative frozen section can be done if any doubt exists.

\section{CONCLUSION}

SGF is a rare entity mostly unknown to surgical community. It is often associated with other congenital anomalies. Resection is not indicated in discontinuous type due to its benign nature. Laparoscopy can be helpful in continuous variety of SGF. Orchidectomy should be avoided in these cases.

\section{REFERENCES}

1. Malik RD, Liu DB. Splenogonadal fusion: an unusual case of an acute scrotum. Reviews in urology. 2013; 15(4):197-201.

2. McPherson F, Frias JL, Spicer D, Opitz JM, Gilbert-Barness EF. Splenogonadal fusion-limb defect syndrome and associated malformations. American Journal of Medical Genetics Part A. 2003 Aug 1; 120(4):518-22.

3. Molaeian M, Shojaei H. Splenogonadal fusion presented with cryptorchidism. Urology Journal. 2009 May 27; 6(2):130-1.

4. Atuan RF, Prades PB, Esgueda AG, de Temiño MR, Sastre BE, Rodruiguez PB, Garcia NA, Pollina JE. Splenogonadal fusion: case report and review of literature. Journal of Pediatric Surgery Case Reports. 2013 Oct 1; 1(10):375-7.
5. Basbug M, Akgun H, Ozgun MT, Turkyilmaz C, Batukan C, Ozcelik B. Prenatal sonographic findings in a fetus with splenogonadal fusion limb defect syndrome. Journal of Clinical Ultrasound. 2009 Jun 1; 37(5):298-301.

6. Kennedy KP, Barnard S, Speakman MJ. Splenogonadal fusion-not just another hydrocoele. The Annals of the Royal College of Surgeons of England. 2006 Mar; 88(2):163-4.

7. Cirillo Jr RL, Coley BD, Binkovitz LA, Jayanthi RV. Sonographic findings in splenogonadal fusion. Pediatric radiology.1999Jan 1;29(1):73-5

8. Sloan JP, Kapila L, Wastie M. Technetium Liver Scan in the Diagnosis of Splenic-gonadal Fusion.British journal of urology. 1987 Apr; 59(4):360-1.

9. Li WF, Luan MX, Ma Z, Chen YJ. Splenogonadal fusion: Report of four cases and review of the literature. Exp Ther Med. 2013 Sep; 6(3):816-818. 2(6):244-48.

\section{The Authors:}

Dr. Muhammad Ali Sheikh

Associate Professor,

Department of Paediatric Surgery,

Shaikh Zayed Hospital, Lahore.

Dr. Fatima Malik

Intern, Department of Paediatric Surgery,

Shaikh Zayed Hospital, Lahore.

Dr. Sadaf Batool

Resident,

Department of Surgical Oncology

Shaukat Khanum Cancer Hospital, Lahore.

Dr. Jamil Akhtar Munir Ahmed

Senior Registrar

Department of Paediatric Surgery,

Shaikh Zayed Hospital, Lahore.

Prof. Tariq Latif

Head of Department Paediatric Surgery,

Shaikh Zayed Hospital, Lahore.

\section{Corresponding Author:}

Dr. Muhammad Ali Sheikh

Associate Professor,

Department of Paediatric Surgery,

Shaikh Zayed Hospital, Lahore.

E-mail: drali444@yahoo.com 
Proceedings, official journal of Shaikh Zayed Medical Complex, Lahore Pakistan is an open access medical journal which publishes research articles in all disciplines of medical sciences. All papers accepted for publication will appear in both print and online.

\section{Scope of journal}

Proceedings will consider for publication human and animal research with implications for human health on all aspects to medical and biological sciences. Research articles on health informatics, health technology, medical education, health economics and management are also considered.

\section{Type of articles}

Original research, Case series, Case reports, Meta-analysis, Letters to editor, Short communications and Clinical Practice Points.

Editor may also invite experts in a relevant field for Special Invited Papers.

\section{Submission and Publication Fees}

Proceedings does not charge author(s) any submission or publication fee.

\section{Provision of printed copies to author(s)}

One complimentary copy is provided to each author of articles published in Proceedings. Additional copies or reprints are available on payment of charges applicable at the time of request.

\section{Manuscript Organization}

Manuscript length should not exceed 2500 words for original research, 3000 words for review articles and meta-analysis, 1500 words for case series and clinical practice points, 500 words for case reports and short communications and 400 words for letter to editor.

Every manuscript should be typed using Microsoft Word using Arial or Calibri font size 12 double spaced on A4 size paper. Each section of manuscript as outlined below should be placed on a separate page.

\section{a. Title page}

Title should be limited to 50 words.

Author names and affiliations (affiliation may be omitted if not associated with any institution or organization at the time of submission)

Corresponding author name and address (include complete postal and an email address, telephone and cellular number including country and area code)

\section{b. Abstract}

For original research articles this section should be structured in to Introduction, Aims and Objectives, Place and Duration of study, Material and Methods, Results and Conclusions. Maximum length of abstract should not exceed 300 words.

At least $3 \mathrm{MeSH}$ terms should be identified at the end of abstract as keywords. This applies to all types of articles.

For all submissions other than original research articles a non-structured abstract not exceeding 250 words with key words should be provided.

\section{c. Main Structure of Research Article}

Introduction: This section supplies sufficient background information for the reader to understand the topic of research and its importance. Only the most salient features with limited references should be included here without exhaustive review of the literature. Research hypothesis and aims and objectives should appear at the end of this section as subheadings.

Material and Methods: Headings are usually not needed for the following details but if needed author can use them.

1. Study design: Mention clearly type of design

2. Study setting/place $\&$ duration

3. Study population

4. Inclusion and exclusion criteria

5. Sample size (input criteria for calculation, non-response rate included or not)

6. Sampling technique and procedure

7. Variables of the study

8. Data collection tool/ questionnaire (validated or self-constructed, If selfconstructed then mention the validity of your tool)

9. Data Collection procedure

- Pilot testing (if done)

- Actual data collection 
10. Data analysis (mention the version of software used).... For details check the detailed guidelines

\section{d. Ethical issues considered and clearance} obtained (Ethics Committee, Institutional Review Board approval number/ ID and certificate on demand of the editor).

\section{e. Statistical Analysis:}

Tests used for representation of data and statistical analysis along with the significance level and software used (if any) should be identified in this section.

\section{f. Results:}

This section should include results of the research or experiment presented in text, tabular or graphic format in a logical sequence. Do not present interpretation of results here. Do not repeat data in text if it has been presented I tables or illustrations and vice versa.

\section{g. Discussion:}

This section should focus on interpretation of the results of the experiment with reference to the reported literature identifying similarities and differences as well as new aspects uncovered with repetition of results.

\section{h. Conclusions:}

Research paper should conclude with the inferences gained from the research.

\section{i. References:}

All references should be in Vancouver style.

Maximum number of references for each type of manuscript are outlined below

Original research $=30$

Review Article, Meta-Analysis and Clinical Practice $=40$

Case reports and case Series $=10$

Short communications and letters to editor $=6$

\section{j. Tables}

Illustrations and Graphs: These should appear on separate pages after references and numbered with Roman numerals (Figure I, Table 1 and so on). Tables should have a title placed at the top while titles for graphs/illustrations should be placed below the respective figure. All digital images should be at least $300 \mathrm{dpi}$ and submitted as JPEG or TIFF format. At the moment only gray scale images are accepted as color printing entails higher costs. Legends if required should be placed below the caption of figures/illustrations. Maximum width for a one column figure is $8.7 \mathrm{cms}$ and for a 2 column figure is $17.8 \mathrm{cms}$. Maximum height including captions is $22 \mathrm{cms}$.

k. Units of measurement:

Only SI units should be used.

\section{Authorship}

Proceedings follows the recommendation of the International Committee of Medical Journal Editors (ICMJE) Recommendations for the Conduct, Reporting, Editing, and Publication of Scholarly Work in Medical Journals available http://www.icmje.org/recommendations/browse/r oles-and-responsibilities/defining-the-role-ofauthors-and-contributors.html.

For a person to be considered an author all four following conditions must be met

1. Substantial contribution in conception, designing, acquisition, analysis or interpretation of data.

2. Manuscript drafting and/or revision for intellectual content

3. Final approval of manuscript

4. Acceptance of accountability for all aspects of research or any questions/queries that might be raised.

Individuals who do not meet all the above 4 criteria but have contributed in the form of funding; general supervision of a research group or general administrative support; and proofreading should be acknowledged at the end of manuscript before the references section either individually or collectively.

Maximum of 8 authors per institution are allowed for each submission.

Manuscripts submitted should clearly document in tabulated form identifying each author and their contribution. This is the Author Contribution Statement and must be signed by all authors.

Sequence of author names that has been submitted with the manuscript cannot be changed subsequently.

Proceedings requires corresponding author whose name appears on the title page to communicate with the journal on behalf of the remaining authors during submission, review, publication and post publication queries.

\section{Conflict of Interest}

All authors are required to provide a signed statement of Conflict Of Interest (COI) if any exists in relation to the article submitted for publication. COI refers to any financial assistance or services received for any aspect of the submitted research. 
This also includes a declaration of financial relationships with any organization for grants, travel bursaries, equipment and administrative support.

\section{Articles not accepted for publication}

A paper submitted to Proceedings will not be processed further if it has been made available in:

- A blog, periodical, or book

- A conference report or proceedings of a symposium

- A technical bulletin or brochure

- Any retrievable source on the internet

\section{Other Resources for manuscript preparation:}

Many free resources are available on internet for language and grammar. Few examples given to help authors;

https://www.grammarly.com, https://www.grammarcheck.net/editor/

\section{Article writing resources:}

- How to Write Articles that Get Published? Jha, K. N. (2014). How to Write Articles that

Get Published. Journal of Clinical and Diagnostic Research : JCDR, 8(9), XG01-

XG03. http://doi.org/10.7860/JCDR/2014/8107.4855 https://www.ncbi.nlm.nih.gov/pmc/articles/PM C4225960/

- 11 steps to structuring a science paper editors will take seriously

A seasoned editor gives advice to get your work published in an international journal, by Angel Borja, $\mathrm{PhD}$.

https://www.elsevier.com/connect/11-steps-tostructuring-a-science-paper-editors-will-takeseriously

\section{Copyright}

All manuscripts published in Proceedings are protected by copyright. Permission to reproduce any material published in Proceedings is required. However, being an open access journal readers may download any number of articles from the web site for their personal non-commercial use.

\section{Plagiarism Policy}

Proceedings takes a very serious view concerning issues related to plagiarism and strictly follows the guidelines provided by HEC (available at www.hec.gov.pk), PMDC (available at www.pmdc.org.pk) and ICMJE (available at www.icmje.org). Failure of author(s) to comply with these guidelines may result in a letter of reprimand and rejection of papers submitted, permanent suspension of privilege to publish in Proceedings, reporting to the affiliated institution or administrative action sought from HEC and/or PMDC depending on the gravity of plagiarism.

\section{For plagiarism checking:}

www.turnitinuk.com/en_gb/features/originalitychec kOR https://www.grammarly.com/plagiarismchecker

\section{Reporting of Trials:}

As of January 2015, Proceedings requires that all trials should be registered with an International RCT Registry. Lists of acceptable trial registries may be accessed at http://www.icmje.org. Manuscripts reporting results of Randomized Control Trials are required to include CONSORT flow diagram available at http://www.consortstatement. org/consort-statement/flow-diagram. Take help of reporting guidelines/ checklists/ flow charts developed for different study designs; examples include, CONSORT for randomized trials, STROBE for observational studies, PRISMA for systematic reviews and meta-analyses, $\underline{\text { STARD }}$ for studies of diagnostic accuracy.

\section{Editorial correspondence:}

All editorial correspondence should be addressed to Dr. Saadia Shahzad Alam Professor of Pharmacology, Shaikh Zayed Postgraduate Medical Institute, Lahore- 54590, Pakistan. For online submission, inquiries and e-mail addresses visit the webpage of Proceedings at:

www.proceedings-szh.com 